certaines zones d'ombre subsistent. Jusqu'à présent, aucune mutation Nf2 n'a été corrélée au CHC chez l'homme. De même, nous n'avons aucune preuve directe chez la souris que la surexpression d'EGFR dans le foie conduise à une prolifération de cellules progénitrices. Il sera donc essentiel à l'avenir de pouvoir établir une analyse comparative des activités EGFR et YAP au sein des mêmes tumeurs hépatiques. On peut néanmoins déjà conclure que cette voie de signalisation semble essentielle au contrôle de la balance entre processus de régénération et processus tumoral hépatique. Gageons qu'elle sera également la prochaine cible d'inhibiteurs pharmacologiques! $\diamond$

Hippo-YAP signaling pathway in the liver: more than a size gatekeeper!

\section{CONFLITS D'INTÉRÊTS}

L'auteur déclare n'avoir aucun conflit d'intérêts concernant les données publiées dans cet article.

\section{RÉFÉRENCES}

1. Zhao B, Li L, Lei Q, Guan KL. The hippo-YAP pathway in organ size control and tumorigenesis: an updated version. Genes Dev 2010 ; 24 : 862-74.

2. Pan D. The hippo signaling pathway in development and cancer. Dev Cell $2010 ; 19: 491-505$.

3. Grusche FA, Richardson HE, Harvey KF. Upstream régulation of the hippo size control pathway. Curr Biol $2010 ; 20$ : R574-82.

4. Song H, Mak KK, Topol L, et al. Mammalian Mstl and Mst2 kinases play essential rôles in organ size control and tumor suppression. Proc Natl Acad Sci USA 2010; $107:$ 1431-6.

5. Zhou D, Conrad C, Xia F, et al. Mstl and Mst2 maintain hepatocyte quiescence and suppress hepatocellular carcinoma development through inactivation of the Yapl oncogene. Cancer Cell 2009; 16:425-38.

6. Zhang N, Bai H, David KK, et al. The Merlin/Nf2 tumor suppressor functions through the YAP oncoprotein to regulate tissue homeostasis in mammals. Dev Cell $2010 ; 19: 27-38$.

7. Lu L, Li Y, Kim SM, et al. Hippo signaling is a potent in vivo growth and tumor suppressor pathway in the

\title{
NOUVELLE
}

\section{Un nouveau type d'horloge circadienne eucaryote}

François-Yves Bouget

L'horloge circadienne: un processus ubiquiste de mesure du temps

Depuis l'aube des temps, les êtres vivants sont exposés aux cycles jour/nuit quotidiens, liés à la rotation de la terre sur elle-même. La plupart des organismes utilisent ces cycles environnementaux pour synchroniser leurs horloges internes qui leur permettent d'ordonner les processus biologiques au cours du cycle jour/nuit comme d'anticiper les changements environnementaux (lumière, température) liés à ce cycle. Ces horloges dites « circadiennes » (de : environ [circa] un jour [dies] en latin) sont des mécanismes précis de mesure du temps qui orchestrent la vie des organismes, des plus petits (cyanobactéries) aux plus grands (champignons, animaux et plantes). C'est, par exemple, le temps de remise à l'heure de cette horloge qui provoque le décalage horaire observé lors de longs voyages en avion. C'est encore la coïncidence entre le temps interne donné par l'horloge à l'organisme et la photopériode (durées relatives du jour et de la nuit qui varient au cours de l'année) qui régule des processus dits annuels, comme la floraison chez les plantes. Chez l'homme, le dérèglement de l'horloge circadienne prédispose à l'apparition de nombreuses maladies comme la dépression saisonnière [12], les cancers et le diabète $[1,2]$. Au cours des dernières années, notre compréhension des mécanismes de l'horloge s'est considérablement accrue grâce à l'étude d'organismes modèles: mouche et souris chez les animaux, arabette chez les plantes, Neurospora chez les mammalian liver. Proc Natl Acad Sci USA 2010 ; 107 : 1437-42.

8. Benhamouche S, Curto M, Saotome I, et al. Nf2/Merlin controls progenitor homeostasis and tumorigenesis in the liver. Genes Dev 2010 ; $24: 1718$-30.

9. Xu MZ, Yao TJ, Lee NP, et al. Yes-associated protein is an independent prognostic marker in hepatocellular carcinoma. Cancer 2009; $115: 4576-85$.

10. Lee KP, Lee JH, Kim TS, et al. The Hippo-Salvador pathway restrains hepatic oval cell prolifération, liver size, and liver tumorigenesis. Proc Natl Acad Sci USA $2010 ; 107: 8248-53$

11. Yi C, Kissil JL. Merlin in organ size control and tumorigenesis: Hippo versus EGFR. Genes Dev 2010 ; 24 : 1673-9.

12. Bardet PL. La voie Hippo contrôle la croissance des organes au cours du développement. Med Sci (Paris) $2009 ; 25: 253-7$.

UPMC Université Paris 6, CNRS, UMR7621

LOMIC, Observatoire océanologique,

F-66651, Banyuls-sur-mer, France.

Fy.bouget@obs-banyuls.fr

champignons ou encore la cyanobactérie Synechococcus pour les procaryotes.

\section{Bases génétiques de l'horloge} circadienne : l'oscillateur transcriptionnel/traductionnel On a longtemps pensé que l'architecture moléculaire des horloges circadiennes reposait exclusivement sur des circuits de gènes composés d'oscillateurs couplés, dont la nature a été révélée par des approches génétiques. Deux gènes $A$ et $B$ permettent à eux seuls de former un oscillateur circadien transcriptionnel/ traductionnel (OTT) simple, où le produit de $A$ (protéine) active la transcription du gène $B$, la protéine $B$ réprimant en retour la transcription de $A$ (Figure 1 , gauche). Ainsi des approches couplées de génomique fonctionnelle et de modé- 


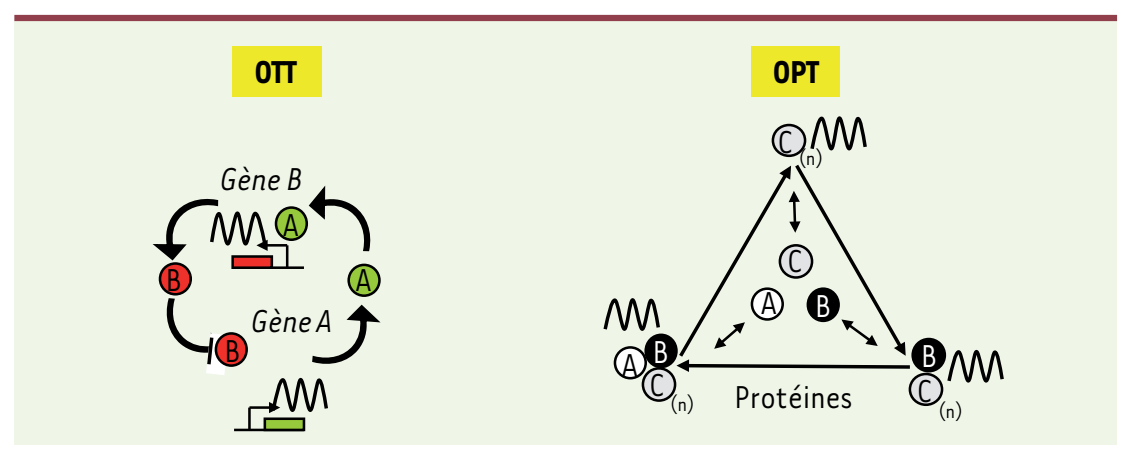

Figure 1. Deux types d'oscillateurs circadiens : l'oscillateur transcriptionnel-traductionnel (0TT) et l'oscillateur post-traductionnel (OPT). Dans l'OTT (à gauche), le rythme résulte de l'activation de la transcription du gène $B$ par la protéine $A$, et de l'inhibition de la transcription du gène $A$ par la protéine B. Dans le seul OPT connu (à droite), décrit chez Synechococcus, 3 protéines A, B et C s'assemblent, se désassemblent et subissent des modifications (phosphorylation de $C$ ) selon un rythme d'environ 24 heures, ceci en l'absence d'activité des gènes.

lisation mathématique chez la microalgue eucaryote Ostreococcus ont montré qu'une boucle de rétrocontrôle négatif composée des deux gènes TOCl (timing of cab expression 1) et CCAl (circadian clock associated 1) permet de rendre compte de la plupart des propriétés de I'horloge circadienne et notamment de sa robustesse aux fluctuations lumineuses de l'environnement [3-6]. Chez les organismes multicellulaires, la situation est bien plus complexe avec l'existence d'horloges couplées dans différents types d'organes et de cellules. De plus, bien que les mécanismes des 0TT soient globalement conservés, les acteurs de cette horloge ne le sont pas entre les grands règnes du vivant.

\section{Les oscillateurs biochimiques ou post-traductionnels}

En plus des circuits de gènes, un oscillateur post-traductionnel (OPT), c'està-dire non génétique, a été caractérisé chez les cyanobactéries. Cet oscillateur biochimique peut être reconstitué par les 3 protéines $K a i^{1} \mathrm{~A}, \mathrm{~B}$ et $\mathrm{C}$ qui, dans un tube, présentent des rythmes de 24 heures d'association/dissociation et de phosphorylation [7] (Figure 1, droite). Deux articles publiés récemment dans la revue Nature rapportent la présence

\footnotetext{
${ }^{1}$ kai signifie rotation ou cycle de nombres, en japonais.
}

d'oscillateurs non génétiques dans le globule rouge humain, cellule énucléée et donc dépourvue d'0TT [8], et dans la microalgue Ostreococcus chez laquelle la transcription du génome cesse rapidement après le passage à l'obscurité [9]. Chez cette microalgue, les rythmes transcriptionnels reprennent lors du passage à la lumière, avec une mémoire du moment du passage à l'obscurité, ce qui indique qu'à l'obscurité une horloge non transcriptionnelle continue de fonctionner. Tant chez l'algue que dans le globule une enzyme-clé dans la détoxification des espèces activées de l'oxygène, est sous le contrôle d'un OPT. De plus, dans le globule rouge, d'autres rythmes circadiens du redox comme celui du NAD(PH) ont été détectés, ce qui pose la question du rôle du redox (et plus généralement du métabolisme qui génère des fluctuations de l'état redox de la cellule) dans l'OPT.

\section{Les interactions de l'OPT}

\section{et de l'0Tा dans l'horloge}

Comment les oscillateurs génétiques et biochimiques interagissent-ils pour engendrer des rythmes circadiens robustes en réponse à des cycles environnementaux ? Chez les cyanobactéries, I'OTT serait un oscillateur esclave de I'OPT [10], néanmoins les rythmes transcriptionnels persistent dans un mutant de rouge, l'oxydation de la peroxyredoxine, phosphorylation de KaiC qui rend I'OPT inopérant [11]. La nature de I'OPT et les interactions 0TT/OPT sont inconnues chez les eucaryotes. Néanmoins, l'analyse de mutants de l'OTT d'Ostreococcus (mutant longue période TOCl:Luc) et de souris (mutant nul CrylCry2) a révélé des anomalies des rythmes d'oxydation de la peroxyredoxine, marqueur de l'activité I'OPT, ce qui suggère des interactions complexes entre ces deux types d'oscillateurs $[8,9]$.

\section{Perspectives}

Au tout début de l'ère moderne d'étude des horloges circadiennes, commencée il y a environ soixante ans, les microalgues eucaryotes se sont avéré être des modèles biologiques de choix du fait de leur simplicité, mais elles furent progressivement délaissées par manque d'outils génétiques au profit d'organismes modèles multicellulaires comme la drosophile ou l'arabette, à l'origine de la découverte des 0TT. La possibilité de développer des approches génétiques et de découpler les mécanismes transcriptionnels et post-traductionnels dans l'obscurité remet sur le devant de la scène des modèles émergents de microalgues comme Ostreococcus pour étudier le couplage des oscillateurs transcriptionnels et post-traductionnels au cœur du métronome circadien de la cellule et retracer l'évolution et la diversification des horloges circadiennes eucaryotes. $\diamond$

A new type of non genetic eukaryotic circadian clock

\section{CONFLIT D'INTÉRÊTS}

L'auteur déclare n'avoir aucun conflit d'intérêts concernant les données publiées dans cet article.

\section{RÉFÉRENCES}

1. Fu L, Lee CC. The circadian clock: pacemaker and tumour suppressor. Nat Rev Cancer $2003 ; 3$ : 350-61.

2. Bass J, Takahashi JS. Circadian integration of metabolism and energetics. Science $2010 ; 330$ : 1349-54.

3. Corellou F, Schwartz C, Motta JP, et al. Clocks in the green lineage: comparative functional analysis of the circadian architecture of the picoeukaryote ostreococcus. Plant Cell $2009 ; 21$ : 3436-49. 
4. Thommen Q, Pfeuty B, Morant PE, et al. Robustness of circadian clocks to daylight fluctuations: hints from the picoeucaryote 0 streococcus tauri. PLoS Comput Biol $2010 ; 6$ : el000990.

5. Troein C, Corellou F, Dixon $L \varepsilon$, et al. Multiple light inputs to a simple clock circuit allow complex biological rhythms. Plant J 2011 (sous presse).

6. Morant $P E$, Thommen $Q$, Pfeuty $B$, et al. A robust two-gene oscillator at the core of Ostreococcus tauri circadian clock. Chaos $2010 ; 20: 045108$.
7. Nakajima M, Imai K, Ito $\mathrm{H}$, et al. Reconstitution of circadian oscillation of cyanobacterial KaiC phosphorylation in vitro. Science 2005 ; 308 : 414-5.

8. O'Neill JS, Reddy AB. Circadian clocks in human red blood cells. Nature 2011 ; 469 : 498-503.

9. O'Neill JS, van Ooijen G, Dixon LE, et al. Circadian rhythms persist without transcription in a eukaryote. Nature 2011 ; 469 : 554-8.

10. Pin X, Byrne M, Xu Y, et al. Coupling of a core posttranslational pacemaker to a slave transcription/ translation feedback loop in a circadian system. PLoS Biol 2010 ; 8 : el 1000394.

11. Kitayama Y, Nishiwaki T, Terauchi K, Kondo T. Dua KaiC-based oscillations constitute the circadian system of cyanobacteria. Genes Dev 2008 ; 22 : 151321

12. Gagné AM, Bouchard G, Tremblay P, et al. Quand la saison devient synonyme de dépression. Med Sci (Paris) $2010 ; 26: 79-82$.

\section{NOUVELle}

\section{La méthylation de I'ADN, un acteur-clé de la pluripotence}

Michaël Weber
Université de Strasbourg/CNRS, UMR 7242, Biotechnologie et signalisation cellulaire, École supérieure de biotechnologie de Strasbourg, boulevard Sébastien Brant, 67412 IIIkirch, France. michael.weber@unistra.fr
$>$ Au cours du développement embryonnaire, les cellules souches pluripotentes perdent leur pluripotence en se spécialisant progressivement en de nombreux types cellulaires aux fonctions variées. Ce processus est contrôlé par des facteurs de transcription qui activent des programmes génétiques spécifiques de chaque lignage cellulaire, mais également par des mécanismes épigénétiques dont des modifications post-traductionnelles des histones et la méthylation de l'ADN. L'ADN est méthylé sur le carbone 5 des cytosines, principalement dans le contexte de dinucléotides $\mathrm{CpG}$, et cette modification est abondante dans le génome de tous les organismes vertébrés. La présence de cytosines méthylées dans la séquence promotrice d'un gène est généralement associée à une répression transcriptionnelle car elle inhibe directement la fixation de certains facteurs de transcription et peut également recruter des répresseurs de la famille des MBP (methyl binding proteins). Puisque les profils de méthylation sont fidèlement copiés au cours de la réplication du génome grâce à l'action de l'enzyme DNMTl (DNA methyltransferase 1), la méthylation de I'ADN peut être considérée comme un système de mémoire épigénétique qui permet de maintenir de manière stable l'état réprimé de certains gènes dans une population cellulaire [1].

\section{Méthylation de gènes maîtres} de la pluripotence au cours de la différenciation des cellules $\varepsilon S$ Plusieurs éléments suggèrent que la méthylation de l'ADN est importante pour la régulation de la pluripotence et des étapes précoces du développement embryonnaire chez les mammifères: (1) les profils de méthylation sont fortement reprogrammés au moment de la restauration de la pluripotence dans les embryons préimplantatoires et les cellules germinales primordiales [1]; (2) les cellules pluripotentes ES (embryonic stem) murines déficientes pour les enzymes DNMT3A et DNMT3B (DNA methyltransferase $3 A$ et $3 B$ ) qui catalysent la méthylation de novo de l'ADN perdent leur capacité de différenciation in vitro [2] ; (3) les souris knock-out pour les gènes Dnmt3a et Dnmt3b meurent à un stade embryonnaire précoce [3]. Pour comprendre le lien entre méthylation de I'ADN et pluripotence, il était dans un premier temps primordial d'identifier les gènes cibles de la méthylation, ce qui a été rendu possible par l'émergence de nouvelles technologies permettant de cartographier la méthylation de l'ADN à grande échelle. Plusieurs équipes de recherche ont ainsi cartographié les promoteurs cibles de la méthylation de l'ADN dans des cellules ES pluripotentes et différenciées. De manière intéressante, ces études ont montré qu'il n'y avait pas de différences de méthylation sur la plupart des gènes entre les cellules pluripotentes et différenciées. $\varepsilon n$ revanche, plusieurs gènes indispensables au maintien la pluripotence comme ceux codant pour 0 ct4, Nanog, Gdf3, Tdgfl, Rexl (mais pas le gène codant pour Sox2) sont des cibles de la méthylation de I'ADN au cours de la différenciation cellulaire [4-6], ce qui suggère que la méthylation de l'ADN participe à la répression du programme de pluripotence au cours $d u$ développement (Figure 1A).

\section{Caractérisation des gènes cibles de la méthylation in vivo}

De nombreux travaux indiquent que les cellules en culture peuvent accumuler des profils de méthylation de l'ADN anormaux. Il était donc important de confirmer in vivo les observations faites dans des systèmes de différenciation in vitro, ce qui est rendu difficile par la faible quantité de matériel biologique qui peut être collecté aux stades embryonnaires précoces. Afin de répondre à cette question, nous avons optimisé la technique de MeDIPl (methylated DNA immuno-

\footnotetext{
${ }^{1}$ Le principe du MeDIP consiste à fragmenter I'ADN par sonication puis faire une immunoprécipitation avec un anticorps qui reconnaît la 5-méthylcytidine. Cette fraction méthylée peut ensuite être hybridée sur n'importe quel type de microarray (repris de [13]).
} 